\title{
Air exposure and sample storage time influence on hydrogen release from tungsten
}

\author{
K.A. Moshkunov ${ }^{a}$, K. Schmid ${ }^{b}$, M. Mayer ${ }^{b}$, V.A. Kurnaev ${ }^{a}$, Yu.M. Gasparyan $^{a}$ \\ ${ }^{a}$ National research nuclear university “MEPhl”, Kashirskoe sh. 31, 115409, Moscow, Russia \\ ${ }^{b}$ Max-Planck-Institut für Plasmaphysik, EURATOM Association, Boltzmannstrasse 2, D-85748 Garching, Germany
}

\begin{abstract}
In investigations of hydrogen retention in first wall components the influence of the conditions of the implanted target storage prior to analysis and the storage time is often neglected. Therefore we have performed a dedicated set of experiments. The release of hydrogen from samples exposed to ambient air after irradiation was compared to samples kept in vacuum. For air-exposed samples significant amounts of $\mathrm{HDO}$ and $\mathrm{D}_{2} \mathrm{O}$ are detected during TDS. Additional experiments have shown that heavy water is formed by recombination of releasing $\mathrm{D}$ and $\mathrm{H}$ atoms with $\mathrm{O}$ on the $\mathrm{W}$ surface. This water formation can alter hydrogen retention results significantly, in particular - for low retention cases. In addition to the influence of ambient air exposure also the influence of storage time in vacuum was investigated. After implantation at $300 \mathrm{~K}$ the samples were stored in vacuum for up to one week during which the retained amount decreased significantly. The subsequently measured TDS spectra showed that D was lost from both the high and low energy peaks during storage at ambient temperature of $300 \mathrm{~K}$. An attempt to simulate this release from both peaks during room temperature storage by TMAP 7 calculations showed that this effect cannot be explained by conventional diffusion/trapping models.
\end{abstract}

Keywords: tungsten, deuterium, hydrogen, retention, time, oxide, heavy water

\section{Introduction}

The conventional way to study hydrogen retention in plasma facing components (PFC's) is to perform a laboratory experiment, where deuterium implantation is done by plasma or ion beam exposure of a sample. Then the amount of retained deuterium is investigated by nuclear reaction analysis (NRA) and thermal desorption spectroscopy (TDS). In the case of tungsten NRA is limited to a maximum depth of $\sim 10 \mu \mathrm{m}$ [1], which means in the case of extensive diffusion (large irradiation time or high temperature) the full amount of deuterium can be detected only by TDS analysis. In most cases $D$ is released in the form of $D_{2}$ and $H D$, however depending on how the experiment is conducted the ion currents of $H D O$ and $\mathrm{D}_{2} \mathrm{O}$ in mass-spectrometer can be considerably larger than $\mathrm{D}_{2}$ and $\mathrm{HD}$ (see Fig. 1). This obscures evaluation of total retained $D$ amount since there is no conventional way to calibrate setup sensitivity for water (mass 19 and 20). Therefore the formation of heavy water should be avoided.

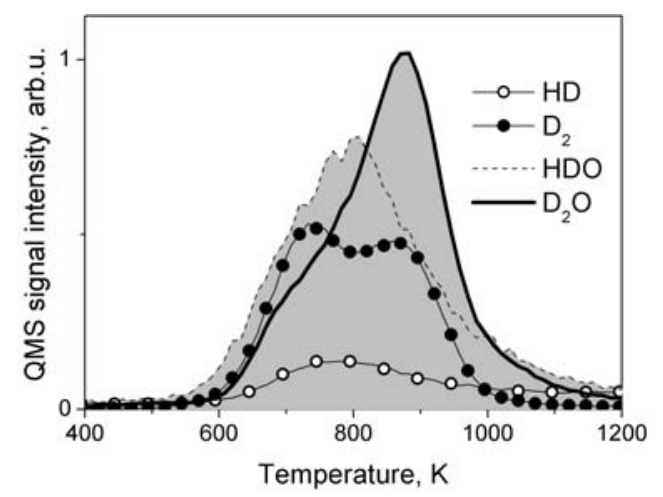

Fig. 1. TDS of the $W$ implanted in plasma with $50 \mathrm{eV} / \mathrm{D}$. Fluence $8 \cdot 10^{23} \mathrm{D} / \mathrm{m}^{2}, 350 \mathrm{~K}$, heating ramp is $2.5 \mathrm{~K} / \mathrm{s}$. 
Another experimental difficulty is that it is almost impossible to keep the time between implantation and analysis the same for all samples even in a frame of a single experiment. It was mentioned in [2] that D inventory with time decreases but no explanation was given. Storage time is never taken into consideration when comparing different experiments. On the other hand, time-dependent release of implanted hydrogen can provide valuable data for the understanding of basic retention mechanisms.

In this paper we will present the results of two sets of experiments: The first part deals with the influence of prolonged air exposure of implanted samples on the measured amount of hydrogen isotopes. In particular the mechanism for heavy water formation during TDS of air exposed deuterium implanted tungsten samples was investigated. The second part deals with time evolution of the hydrogen inventory during storage of implanted samples in vacuum. These experiments focus on the e-folding time of deuterium release during room temperature in vacuum storage and on how it is released from the individual trap sites that have been identified in tungsten [2].

\section{Experimental}

For the experiments conducted in IPP the tungsten (W) samples were cut from Plansee AG powder-metallurgy W-rod, polished mechanically, sonicated in ethanol and annealed at $1250 \mathrm{~K}$ for $15 \mathrm{~h}$ to release residual stress and impurities. The irradiation of the samples with deuterium ions was performed in the PLAQ setup, which is described in [3] and in the High current ion source at IPP [4], which is a mass-filtered ion beam implantation device.

PLAQ is a remote ECR plasma device, with RF applied to a target holder to obtain the desired bias and enhance plasma density. The plasma was operated with a $D_{2}$ gas pressure of $1 \mathrm{~Pa}$ which results in $\mathrm{D}_{3}{ }^{+}$being the dominant ion species. Thus with an applied bias of $450 \mathrm{~V}$ the average energy becomes $150 \mathrm{eV} / \mathrm{D}$ with the flux of approximately $6 \cdot 10^{19} \mathrm{D} /\left(\mathrm{m}^{2} \cdot \mathrm{s}\right)$. The sample holder can be cooled or heated and temperature is measured via an IR camera. Several samples can be mounted on the sample holder to ensure that all of them are exposed to the same irradiation conditions. After irradiation the samples are moved to the TESS TDS machine [5]. There they are stored in the glass tube TDS setup, in vacuum during the entire experiment. They can be moved inside the tube one by one to the heated area without breaking the vacuum. Heating is provided by an external oven, in which the quartz tube is placed, with a heating ramp rate varying from 0.5 to $0.8 \mathrm{~K} / \mathrm{s}$ (see the spectra).

To perform TDS measurements after implantation without air exposure the samples were implanted at the High current ion source at IPP [4]. This is a mass-filtered ion beam implanter with deceleration optics. $\mathrm{A} \mathrm{D}_{3}{ }^{+}$ion beam was used with an energy of $200 \mathrm{eV} / \mathrm{D}$ and average fluence of $4 \cdot 10^{18} \mathrm{D} /\left(\mathrm{m}^{2} \mathrm{~s}\right)$. After implantation the sample is transferred in vacuo to a dedicated TDS chamber. For TDS the sample is heated from the unirradiated side by an electron beam such that the sample temperature follows a linear temperature ramp of $1.5 \mathrm{~K} / \mathrm{s}$. The temperature is measured by a $\mathrm{K}$-type thermocouple, attached to the sample. In the experiment without air exposure vacuum was lower than $10^{-9} \mathrm{mbar}$ and according to residual gas analysis oxygen and water levels were below $10^{-10}$ mbar, so that the significant oxidation of tungsten is not likely to occur during vacuum storage.

To further investigate the release of implanted deuterium during storage a special sample pair was produced. Two identical W samples were implanted in PLAQ then immediately after implantation one of the samples was coated with aluminum oxide to keep $D$ from diffusing out of the surface. The aluminum oxide film was deposited in a filtered arc discharge setup at IPP, which is described elsewhere [6]. Both samples were stored in air for 4 month before the TDS analysis was performed in high resolution TDS setup in MEPhI, Moscow described in [7]. In this setup target is radiatively heated with constant rate of $2 \mathrm{~K} / \mathrm{sec}$.

Another TDS study with this set-up was performed for a batch of $W$ samples irradiated in the ion-beam monochromator, similar to HSQ. The irradiation was performed at RT, with $\mathrm{D}_{3}{ }^{+} 10 \mathrm{keV}$ ions to fluencies of $1.4 \cdot 10^{22} \mathrm{D} / \mathrm{m}^{2}$. Between irradiation and TDS samples were stored in air.

The $D$ release from the TDS spectra was calculated as in equation 1:

$R_{D}=K_{D_{2}}\left(2 \cdot I_{D_{2}}+K_{H D} / K_{D_{2}} \cdot I_{H D}\right),(1)$

where $I_{D 2}$ and $I_{H D}$ are the quadrupole signal intensities for mass 3 and $4, K_{D 2}$ and $K_{H D}$ are the corresponding sensitivities. $K_{H D}$ was determined as an average of directly measured $K_{D 2}$ and $K_{H 2}$ and is equal to 0.7 in HSQ device and 0.5 for the Moscow TDS stand.

\section{Heavy water formation study results}


In the High current ion source one W sample was sputter-cleaned and annealed in vacuum. Without breaking the vacuum sample was then irradiated by $200 \mathrm{eV} / \mathrm{D}$ at RT up to fluence $1.5 \cdot 10^{23} \mathrm{D} / \mathrm{m}^{2}$. Immediately after the implantation the sample was transferred in vacuo to the dedicated TDS chamber. There it was stored for $24 \mathrm{~h}$ in vacuum and then TDS was performed. The second $\mathrm{W}$ sample was treated almost the same way except that it was stored for $24 \mathrm{~h}$ in ambient air. The resulting spectra for both samples are presented in Fig. 2.

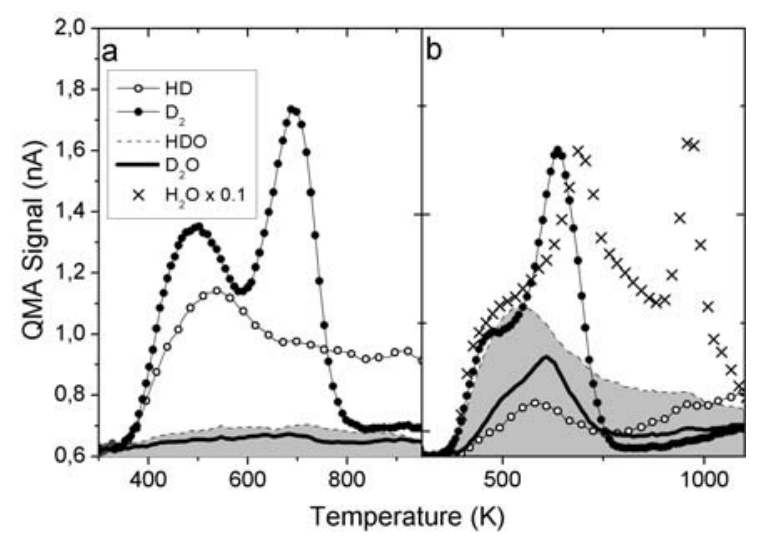

Fig. 2. TDS spectra for irradiated $W$ stored in a) vacuum and b) air. In a) $\mathrm{H}_{2} \mathrm{O} 0.1$ signal lies below the lower boundary.

For the air exposed sample the first peak for $H D$ and $D_{2}$ is reduced and the heavy water signals are much higher compared to the vacuum stored sample. Additionally, D release estimation based on integration of HD and $D_{2}$ signals shows that $\sim 17 \%$ less $D$ released in the case of air exposure compared to vacuum storage. The lacking $D$ could be lost through $\mathrm{HDO}$ and $\mathrm{D}_{2} \mathrm{O}$, but we have no sensitivity factors for these species. Three mechanisms can be proposed to explain heavy water presence during TDS. The first is an isotopic exchange of $D$ with $\mathrm{H}_{2} \mathrm{O}$ in the residual gas (this can happen on the sample surface, chamber walls or hot parts), the second is that $\mathrm{HDO}$ and $\mathrm{D}_{2} \mathrm{O}$ can be brought in TDS chamber with the sample. Finally the third possibility is that heavy water can be formed by recombination of deuterium, hydrogen and oxygen on the surface during TDS. The hydrogen thereby originates from the background gas or comes from a sample and oxygen comes from the natural $\mathrm{W}$ oxide.

To check the first mechanism the following experiment was performed in the TESS main chamber. A heated W sample was exposed to a gas atmosphere (low pressure $<10^{-4} \mathrm{~Pa}$ ) with varying amounts of $\mathrm{D}_{2}$ and $\mathrm{H}_{2} \mathrm{O}$. The pressures of $\mathrm{D}_{2}$ and $\mathrm{H}_{2} \mathrm{O}$ and the sample temperature were varied to probe the impact of these three parameters on the formation of heavy water. Results of the investigation are presented in Fig. 3.

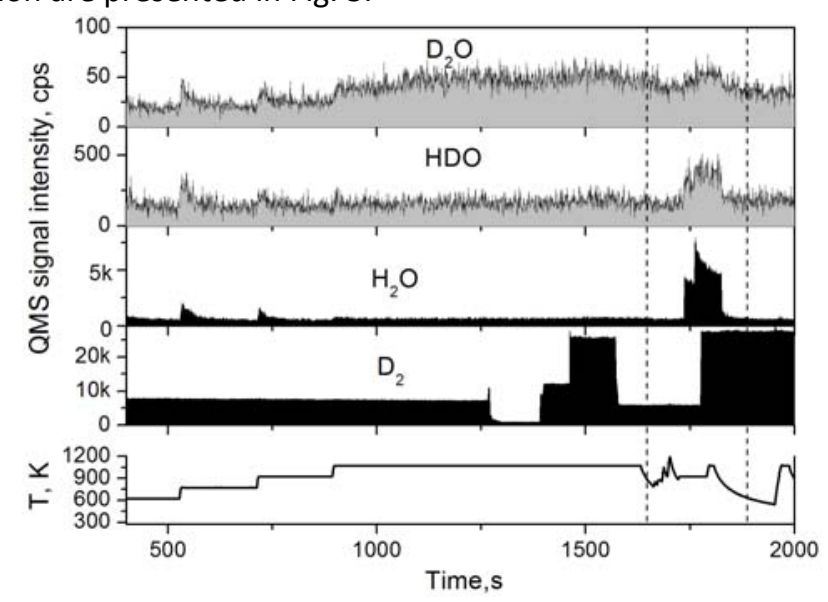

Fig. 3. Time dependence of $W$ sample temperature, and QMS signal intensity for $\mathrm{D}_{2}, \mathrm{H}_{2} \mathrm{O}, \mathrm{HDO}$ and $\mathrm{D}_{2} \mathrm{O}$.

The variation of temperature, $D_{2}$ and $\mathrm{H}_{2} \mathrm{O}$ pressures do not result in $\mathrm{D}_{2} \mathrm{O}$ intensity change higher than background fluctuations, which is especially visible in the dashed lines limited region. The $\mathrm{HDO}$ to $\mathrm{H}_{2} \mathrm{O}$ signal ratio is smaller compared to the ratio observed for the sample (fig. $2 b$ ). Additionaly in the fig. $2 \mathrm{~b}$ the HDO release does not correlate with $\mathrm{H}_{2} \mathrm{O}$ 
release as it does in fig. 2. $\mathrm{D}_{2} \mathrm{O}$ to $\mathrm{H}_{2} \mathrm{O}$ ratio in fig. 3 is much smaller compared to fig. $2 b$ despite the amount of $\mathrm{D}_{2}$ in fig. 3 being much higher than in fig. $2 b$.

Another experiment was done in the TESS main chamber to test whether HDO and $\mathrm{D}_{2} \mathrm{O}$ are adsorbed on the sample prior to TDS. A W sample implanted with D at $450 \mathrm{~K}$ was placed on the $450 \mathrm{~K}$ preheated sample holder to desorb all the water from its surface. As shown in Fig. 4, where the arrow indicates the moment when the sample was placed on the holder, there is no heavy water desorption detected.

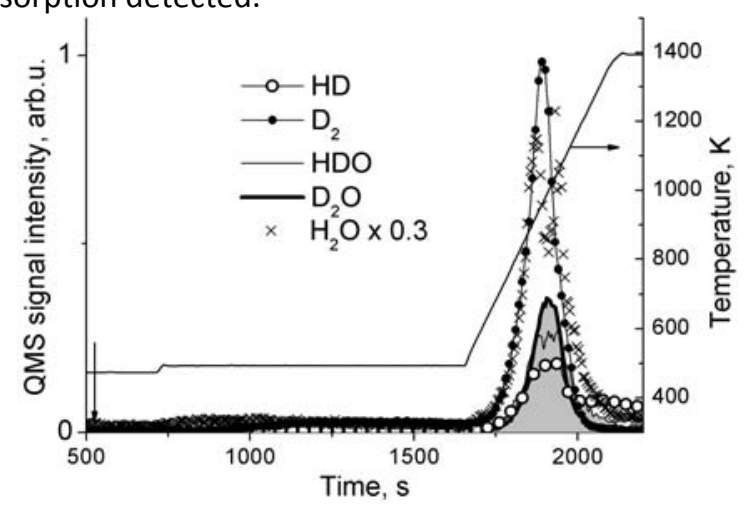

Fig. 4. TDS with preheating of the $W$ implanted in plasma with $150 \mathrm{eV} / \mathrm{D}$. Fluence $8 \cdot 10^{23} \mathrm{D} / \mathrm{m}^{2}, 450^{\circ} \mathrm{K}$, heating ramp is $2.5 \mathrm{~K} / \mathrm{s}$.

However after some time the heat ramp was turned on and heavy water appeared in the spectrum. This finally rules out the second mechanism since this implies that $\mathrm{HDO}$ and $\mathrm{D}_{2} \mathrm{O}$ are not intrinsic impurities adsorbed on the sample since it would have desorbed during preheating and are not produced by an isotopic exchange outside the sample, as shown previously.

So we are driven to conclusion that observed heavy water desorption happens due to recombination of $D$ and $H$ with oxygen from surface $\mathrm{W}$-oxide. This oxide is mainly formed during air exposure.

\section{Storage time influence investigation results}

D implantation for the time-release study in the PLAQ plasma device was done at RT and $500 \mathrm{~K}$ with $150 \mathrm{eV} / \mathrm{D}$ up to fluence $10^{24} \mathrm{D} / \mathrm{m}^{2}$. Six simultaneously irradiated $\mathrm{W}$ samples were transferred through air to the TESS tube TDS and placed in the pumped quartz tube. The transfer took less the 10 minutes which from our previous experiments is known not to lead to massive oxidation of the $\mathrm{W}$ substrate and does thus not lead to problems with heavy water formation.

After the transfer TDS analysis was performed for these samples after different storage times, covering the 15-140 $\mathrm{h}$ range. In Fig. 5 typical TDS spectra for RT and $500 \mathrm{~K}$ implantations are presented.

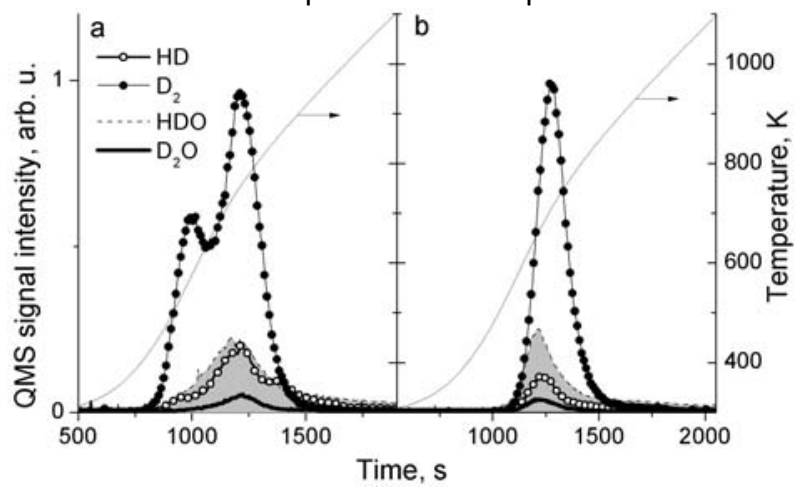

Fig. 5. TDS spectra for a) RT and b) $500^{\circ} \mathrm{K}$ irradiation with $150 \mathrm{eV} / \mathrm{D}$. Fluence $10^{24} \mathrm{D} / \mathrm{m}^{2}$.

Heavy water signals are low compared to $D_{2}$ and HD, allowing us to calculate the total amount of released $D$, which is plotted in Fig. 6 a as a function of storage time. 


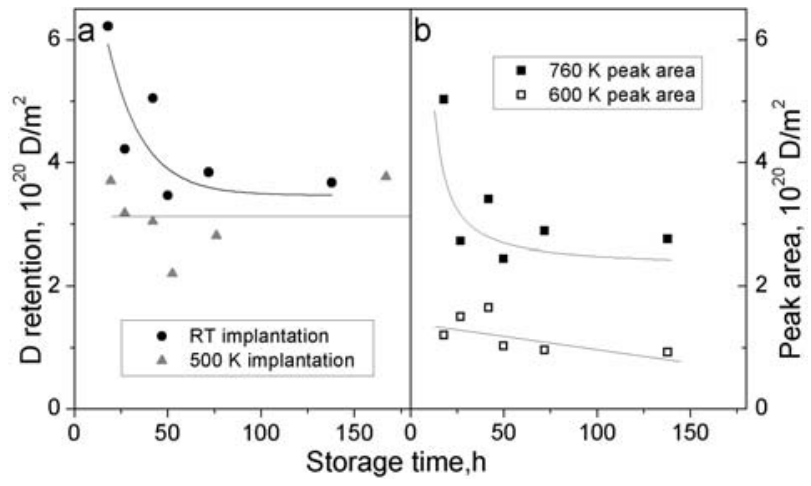

Fig. 6. Plotted as a function of the storage time: a) amount of $D$ released in TDS and b) areas of TDS peaks (lines are guide to the eye).

The TDS spectra for the $500 \mathrm{~K}$ can be perfectly fitted by two Gaussian peaks, around 600 and $760 \mathrm{~K}$. The so obtained peak areas are plotted in Fig. $6 \mathrm{~b}$ as function of storage time. The most striking feature is that the high temperature peak decreased most during room temperature storage which is unexpected for the high energy traps. In an attempt to explain these dependencies, TMAP7 was used to model the underlying processes. Firstly, the TDS of RT irradiated W was reproduced with detrapping energies $E_{t}$ of 1.4 and $1.8 \mathrm{eV}$, diffusion coefficient of $D=4 \cdot 10^{-7} \cdot \exp (-0.4 \mathrm{eV} / T)$, unlimited recombination and realistic temperature ramp. These trapping and diffusion parameter were taken from literature [10] and yielded an acceptable agreement with the experimental data as can be seen in Fig. 7a. Traps were homogeneously distributed over first $5 \mu \mathrm{m}$ of the sample and as calculations confirmed the depth profile of the traps does not influence significantly on the time-dependent release for $E_{t}>1 \mathrm{eV}$.

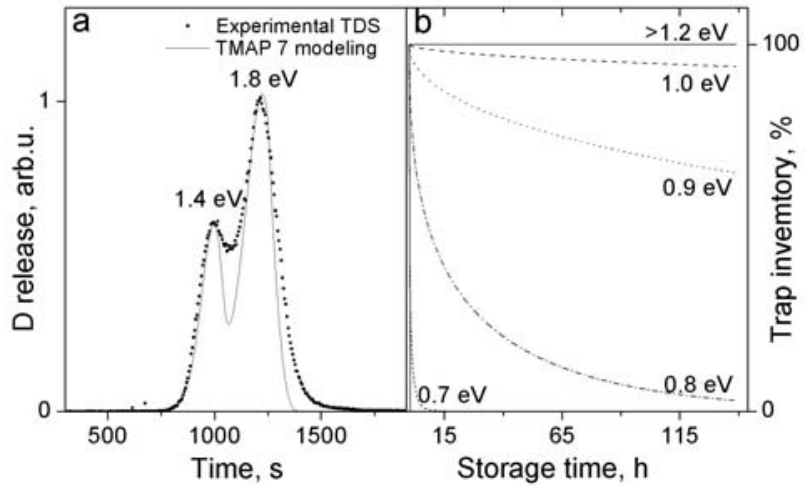

Fig. 7. TMAP modeling results for: a) TDS of RT irradiated $W$ and b) trap inventory for different detrapping energies as a function of storage time at RT.

Then the $D$ trap inventory (or retention) reduction during room temperature storage with time was modeled using the same parameters except for trapping. Only one trap was modeled and its trap energy $E_{t}$ was varied from 0.7 to $1.2 \mathrm{eV}$ and the results are shown in Fig. $7 \mathrm{~b}$. It is obvious that traps with $E_{t}>1.2 \mathrm{eV}$ do not desorb $\mathrm{D}$ during room temperature storage. Variation of diffusion coefficient or trap density distribution in the reasonable limits does not influence the resulting TDS spectra for $1.2 \mathrm{eV}$ and higher energy traps significantly since the release at the observed peak temperatures is governed by the detrapping. So the experimental observation appears to be completely inconsistent with the model implemented in TMAP. This drives us to the conclusion that there is some other mechanism responsible for the $D$ release with time.

The slow decay with time of the amount of $D$ released from sample irradiated at $500 \mathrm{~K}$ compared to the release from RT irradiated sample can be explained by that the $D$ release speed at fixed temperature is a nonlinear function of $D$ concentration in the sample.

To check the ultimate consequences of the time-dependent release the following experiment was done. Two identical W samples were irradiated at RT in PLAQ with $150 \mathrm{eV} / \mathrm{D}$ up to fluence $10^{24} \mathrm{D} / \mathrm{m}^{2}$. Then one of them was coated by thin $(\sim 40 \mathrm{~nm})$ amorphous $\mathrm{Al}_{2} \mathrm{O}_{3}$ film. This was done to seal the implanted $\mathrm{D}$ in W, preventing it from releasing since $\mathrm{Al}_{2} \mathrm{O}_{3}$ is a hydrogen diffusion barrier. After 4 month of in-air storage they were analyzed by TDS in the high resolution TDS machine at MEPhl. The measured $D$ release calculated from the $D_{2}$ and HD signals is presented in Fig. 8 . 


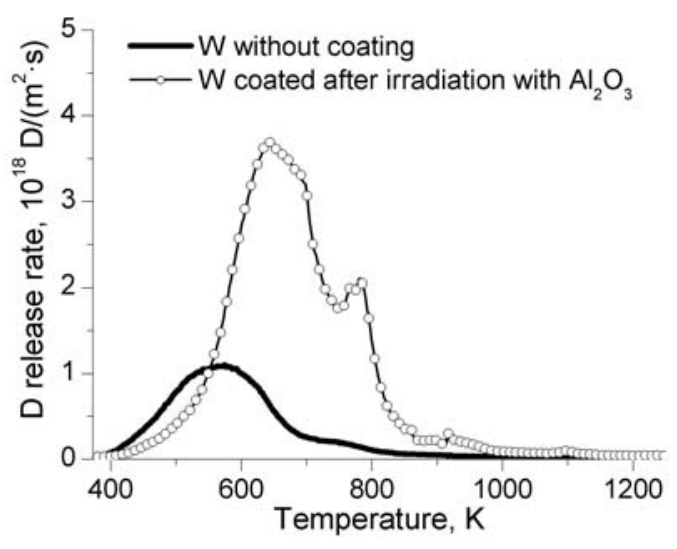

Fig. 8. $D$ release for identically irradiated $W$ samples after 4 month of storage.

As can be seen from fig. 8 the uncoated $W$ sample lost $66 \%$ of the implanted $D$ compared to the coated one. This result can be seen as an upper limit for the possible loss of implanted $D$ during long term storage.

The $D$ high-energy trap inventory decrease in $W$ was observed also in experiments performed with ion beam implantation set-up in MEPhl. TDS analysis results for $\mathrm{W}$ samples $(0.5 \mathrm{~mm}$ ITER-grade tungsten) irradiated with a fluence of $1.4 \cdot 10^{22} \mathrm{D} / \mathrm{m}^{2}$ of deuterium ions are presented in Fig. 9 for storage times ranging from 3 hours to 3 month. The decrease of both low- and high-temperature peaks is obvious. The reduction of the $D$ inventory after 3 month storage compared to 3 hours is $61 \%$ that coincides with the previously described experiment.

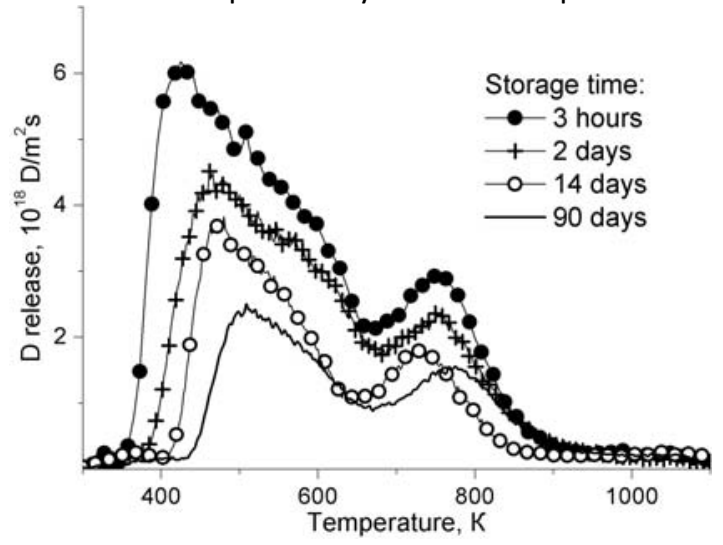

Fig. 9. D release from W samples implanted by $10 \mathrm{keV} \mathrm{D}_{3}{ }^{+}$at RT with fluence $1.4 \cdot 10^{22} \mathrm{D} / \mathrm{m}^{2}$ for storage times of 3 hours, 2 , 14 and 90 days.

That implies that $\mathrm{Al}_{2} \mathrm{O}_{3}$ film coating can be applied to preserve the hydrogen inventory from being released during storage. This technique can be applied in situations when storage time for the samples varies a lot. This coating alternates the shape of TDS spectra (fig. 8) but maintains the total hydrogen amount.

\section{Conclusion}

In this paper the influence of storage conditions and duration of storage of $D$ implanted $W$ samples on the $D$ retention and release was investigated.

It was found that the source of heavy water formation during TDS analysis of $D$ implanted tungsten was a recombination of $\mathrm{O}$ from surface oxide with releasing $\mathrm{D}$ and $\mathrm{H}$. Therefore long term air exposure (in the order of 1 day) must be avoided for accurate measurements of retention via TDS since the release of $D$ in heavy water cannot be quantitatively measured TDS due to calibration obstacles.

A TDS study of the storage time dependent release of $D$ from $W$ revealed that the amount of trapped $D$ inventory in both the high and low energy traps is decreasing with time. This release goes faster than it can be expected from modeling done by TMAP 7. Therefore, one should suggest some specific channels of release to describe that. 
A new technique for sample storage was developed based on aluminum oxide coating. It allows excluding storage time influence on retention measurements.

Acknowledgments

This work is supported by Hemholtz-Russian Joint Research Group. We also thank Alexander Rusinov and Gabriele Matern for experimental assistance.

Literature

[1]. M. Mayer, E. Gauthier, K. Sugiyama, U. von Toussaint. Nucl. Instr. and Meth. B 267 (2009) 506-512

[2] O. V. Ogorodnikova et. al. , J. appl. Phys., 103, (2008) 034902

[3]. B. Landkammer, A. von Keudell, W. Jacob, J. Nucl. Mater. 264 (1999) 4855.

[4] J. Roth, J. Bohdansky, W. Ottenberger, Max-Planck-Institut für Plasmaphysik, Technical report IPP 9/26, Garching, 1979.

[5] E. Salancon, T. Dürbeck, T. Schwarz-Selinger, F. Genoese, W. Jacob, J. of Nucl. Mat. 376 (2008) 160-168

[6]. R. Brill et. al., Surf. Coat. Technol. 174-175 (2003) 606.

[7]. A. Pisarev et. al., J. Nucl. Mater. 390-391 (2009) 677. 